\title{
Morphological evolution of cluster galaxies
}

\author{
C. Mastropietro, B. Moore, J. Diemand, L. Mayer and J. Stadel \\ Institute for Theoretical Physics, University of Zürich, CH-8057 Zurich, Switzerland, \\ e-mail: chiara@physik.unizh.ch
}

\begin{abstract}
The recent discovery of hidden non-axysimmetric and disk-like structures in bright Virgo dwarf elliptical and lenticular galaxies indicates that they may have late-type progenitors. Using high resolution N-body simulations we follow the evolution of disk galaxies within a $\Lambda \mathrm{CDM}$ cluster simulated with $10^{7}$ particles where the hierarchical growth and galaxy harassment are modelled self-consistently. Most of the galaxies undergo significant morphological transformation, even at the outskirts of the cluster, and move through the Hubble sequence from disks to spheroidals. The time evolution of the simulated galaxies is compared with unsharp-masked images from the VLT and we compare the kinematics of our models with the latest high resolution spectroscopic studies from the Keck telescope. We will also discuss the properties of diffuse intra-cluster light formed by tidal debris stripped away from the infalling disks by gravitational interactions.
\end{abstract}

\section{Introduction}

Early type dwarfs ( $\mathrm{dE}$ and $\mathrm{dSO}$ ) are the most common type of galaxies in the nearby universe, yet their origin and nature are still unknown. Formation scenarios include mainly two different hypotheses: 1) dEs and dSOs are primordial galaxies, 2) they are the result of a morphological transformation of spiral and irregular galaxies accreting into the cluster. This latter idea is supported through observational evidence, i.e. the relative number density of early type galaxies increases with the local galaxy density, suggesting that the enviroment drives galaxy evolution. Moore, Lake \& Katz (1998) have shown that galaxy harassment in clusters transforms spirals into spheroidals. Harassment can explain the morphological evolution of the small spiral and irregular galaxies observed in clusters at redshift $z \sim 0.4$ (Dressler et al. 1984) into dwarf ellipticals in the nearby universe (Moore et al. 1996). Moreover the radial velocities of early type galaxies in the Virgo cluster seem to indicate that these galaxies are not an old cluster population (Conselice, Gallagher \& Wyse 2001) but originate from infalling field galaxies. According to this evolutionary scenario we expect to find nearby cluster galaxies that are currently undergoing morphological transformation and retain part of their disk nature. Recent observations from VLT and Keck telescope confirm that early type galaxies have a broad range of photometric and kinematical characteristics. In particular the degree of rotational support is found to vary from zero to a value close to one expected for a galaxy flattened by rotation (De Rijcke et al. 2001, Simien \& Prugniel 2002, Pedraz et al. 2002, Geha et al. 2002, 2003). Jerjen, Kalnajs \& Binggeli (2000) and Barazza, Binggeli \& Jerjen (2002) discovered hidden spiral structures and bar features in five bright dEs in the Virgo cluster, concluding that the fraction of early type dwarfs hosting a disk component could be larger than 20\%. Spirals and disks were also observed within dwarf spheroidal galaxies in the Coma (Graham, Jerjen \& Guzmán 2003) and Fornax clusters (De Rijcke et al. 2003). 
The aim of this work is to follow the evolution of disk galaxies orbiting in a cluster enviroment using high resolution N-body simulations and to compare the final harassed remnants with the latest photometric and spectroscopic data.

\section{Simulations}

All the simulations have been carried out using PKDGRAV (Stadel 2001). The galaxy models are multi-component systems with an exponential stellar disk embedded in a dark matter halo and were built using the technique described by Hernquist (1993). The density profile of the NFW halo is adiabatically contracted in response to baryonic infall (Springel \& White 1999). Each galaxy is modelled with $10^{6}$ particles, of which $10^{5}$ are in the disk. The gravitational softening is set to $0.5 \mathrm{kpc}$ for the dark halo and $0.1 \mathrm{kpc}$ for disk particles. The galaxies are low surface brightness models with a central surface brightness $\mu_{B}=22 \mathrm{mag} \operatorname{arcsec}^{-2}$, assuming a B-band mass to light ratio $\simeq 3$. The peak circular velocity is $100 \mathrm{~km} \mathrm{~s}^{-1}$, the total mass and stellar mass are respectively $7 \times 10^{10} \mathrm{M}_{\odot}$ and $4.2 \times 10^{9} \mathrm{M}_{\odot}$ while the initial disk scale length is $1.5 \mathrm{kpc}$ at $z=0$. The Toomre stability parameter $\mathrm{Q}$ was set to 1.5 at the disk scale length. A cluster with a mass comparable to Virgo $\left(M_{v i r}=3.1 \times 10^{14}\right)$, at $z=0$ and a virial radius of $1.8 \mathrm{Mpc}$ was selected from a $300 \mathrm{Mpc} \Lambda \mathrm{CDM}$ cube simulation and resimulated at high resolution (Diemand, Moore \& Stadel 2004b). This halo corresponds to the intermediate resolution cluster D9 of Diemand, Moore \& Stadel (2004a). The number of particles within the virial radius is $\sim 6 \times 10^{6}$, while the softening length is $2.4 \mathrm{kpc}$. At $z=0.5$ we replaced 20 random cluster particles (10 within the virial radius and 10 within $20 \%$ of the virial radius) with the high resolution galaxy models. Disk scale lengths and internal velocities were rescaled according to the redshift and cosmology.

\section{Results}

The evolution of the galaxies within the cluster is quite violent and is due to a sequence of strong gravitational encounters with substructures and the global cluster potential. Most of the central galaxies lose a significative fraction of stars and undergo a complete morphological transformation from disks to spheroidal systems. Figure 1 represents the different phases of the evolution of a galaxy transforming in a dE. The images are obtained from the simulations applying the unsharp masking technique described in Barazza et al. (2002), in order to highlight the presence of hidden structures. The first stage of the evolution is characterised by the formation of a strong bar and an open spiral pattern in the disk. The spiral arms are easily destroyed while the remaining material forms a ring structure around the bar. The bar+ring phase is quite stable and is apparent in the final state of several of the remnant galaxies. Often the ring is not visible directly in the simulation output without applying the unsharp masking. When gravitational heating removes the remaining spiral and ring features, only a naked bar remains. The bar usually buckles, and as a result the central part of the galaxy becomes more spherical; the buckled bar is also subject to tidal heating and loses mass from its edges. Table 1 shows the main characteristics of the harassed remnants within the virial radius of the cluster. The final state is always quite prolate, with the exception of the first two galaxies which are roughly spherical. This is not surprising since most of the galaxies retain part of the radial anisotropy that originated during the bar phase (Mayer et al. 2001). There is a clear correlation between the c/a ratio, which indicates the flattening of the disk, and the final stellar mass. As shown in the top left plot in Figure 3, massive stellar remnants have smaller c/a values and retain more of their initial disk nature, while galaxies that have lost most of their stars tend to be more spheroidal. On average 


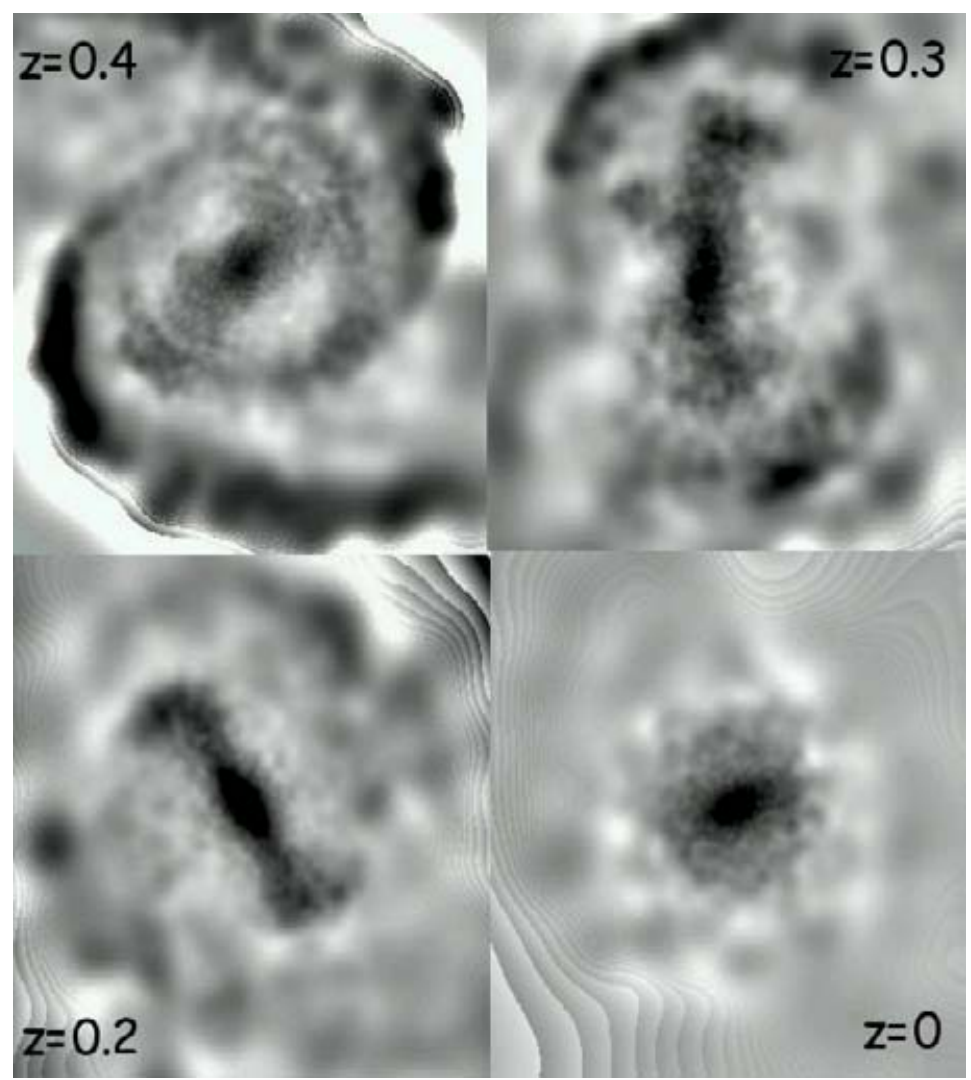

Figure 1. Morphological transformation of a disk galaxy into a dwarf elliptical. For each frame the redshift is indicated.

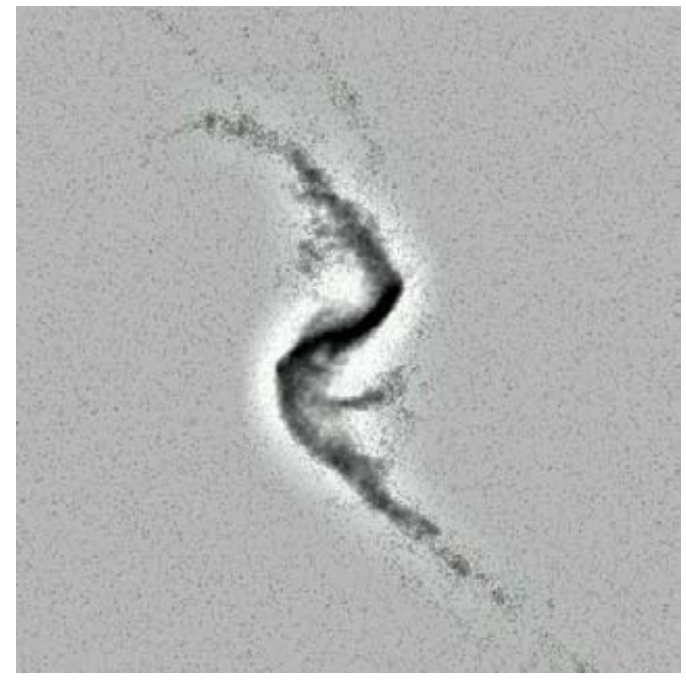

Figure 2. Tidal tails generated during a pericentric passage. The time scale of these smooth features is quite short (tipically about $0.2-0.3$ Gyr) since they are perturbed by close encounters with cluster substructures. 


\begin{tabular}{cccccc}
\hline$r_{t}(\mathrm{kpc})$ & $r_{e}(\mathrm{kpc})$ & $M_{d}\left(10^{9} M_{\odot}\right)$ & $M_{s}\left(10^{9} M_{\odot}\right)$ & $c / a$ & $b / a$ \\
\hline 4.9 & 0.6 & 2.3 & 0.5 & 0.8 & 0.7 \\
7.3 & 0.9 & 8.0 & 1.5 & 0.8 & 0.8 \\
12.3 & 1.1 & 10.9 & 1.7 & 0.5 & 0.4 \\
14.0 & 1.1 & 16.1 & 1.8 & 0.5 & 0.4 \\
8.9 & 1.3 & 5.5 & 2.1 & 0.7 & 0.5 \\
13.7 & 1.3 & 9.7 & 2.4 & 0.6 & 0.5 \\
15.8 & 1.4 & 23.4 & 2.6 & 0.6 & 0.5 \\
11.6 & 1.7 & 9.4 & 3.1 & 0.6 & 0.4 \\
33.0 & 1.9 & 18.0 & 3.5 & 0.4 & 0.4 \\
12.4 & 2.7 & 19.2 & 3.9 & 0.3 & 0.2 \\
33.9 & 3.2 & 15.9 & 4.0 & 0.5 & 0.3 \\
18.9 & 2.4 & 19.0 & 4.0 & 0.5 & 0.3 \\
\hline
\end{tabular}

Table 1. Final state of the remnants that lie within the virial radius of the cluster at $z=0$. For each galaxy we list tidal $r_{t}$ and effective $r_{e}$ radius, dark matter $M_{d}$ and stellar $M_{s}$ mass. The tidal radius is calculated balancing kinetic and potential energy in spherical shells centered on the remnant, while $r_{e}$ is the radius containing half of the light. The last two columns indicate the average axial ratios (respectively short and intermediate axis to major axis) measured within $r_{e}$.

more than $40 \%$ of the stars are lost forming irregular tails of low surface brightness $\left(\mu_{B}<30\right.$ mag $\left.\operatorname{arcsec}^{-2}\right)$ tidal debris. The next two plots in Figure 3 represent the surface brightness and velocity dispersion profiles of the remnants compared to the initial values. Although a significative fraction of the stellar mass has been stripped from the disks, the central surface brightness increases by 1-2 mag $\operatorname{arcsec}^{-2}$ as result of the increase in the stellar random motions and conservation of phase space (Moore et al. 1999). Within $r_{e}$ the remnants still have exponential profiles with scale lengths in the range $0.5-1.0 \mathrm{kpc}$. The radial velocity dispersion rises due to bar formation and impulsive shocks. The degree of rotational support of the two spherical remnants is close to zero (bottom right plot), while the $v / \sigma$ ratio of the other galaxies depends on the viewing angle. In particular, if viewed down along the short axis, which is always the rotational axis, the galaxies show a $v / \sigma \sim 0$. Along the major axis they have the maximum rotational support, while the intermediate axis projection produces the largest ellipticity values.

\section{Conclusions}

We have shown that a significant fraction of cluster galaxies undergo a morphological transformation from pure disk systems to dEs and dSOs with photometric and kinematical characteristics similar to observed galaxies. The degree of this transformation is directly correlated with the loss of mass. Up to $80 \%$ of the stellar mass is lost due to tidal effects and forms a diffuse low surface brightness distribution of irregular tidal streams.

\section{References}

Barazza, F. D., Binggeli, B. \& Jerjen, H. 2002 A\&A 391, 823

Conselice, C. J., Gallagher, J. S. \& Wyse, R. F. G. 2001 ApJ 559, 791

De Rijcke, S., Dejonghe, H., Zeilinger, W. W. \& Hau, G. K. T. 2001 ApJ 559, L21

De Rijcke, S., Dejonghe, H., Zeilinger, W. W. \& Hau, G. K. T. 2003 A\& A 400, 119

Diemand, J., Moore, B. \& Stadel, J. 2004a astroph 0402267, MNRAS submitted

Diemand, J., Moore, B. \& Stadel, J. 2004b astroph 0402160, MNRAS submitted

Dressler, A., Oemler, A. J., Butcher, H. R. \& Gunn, J. E. 1994 ApJ 430, 107 

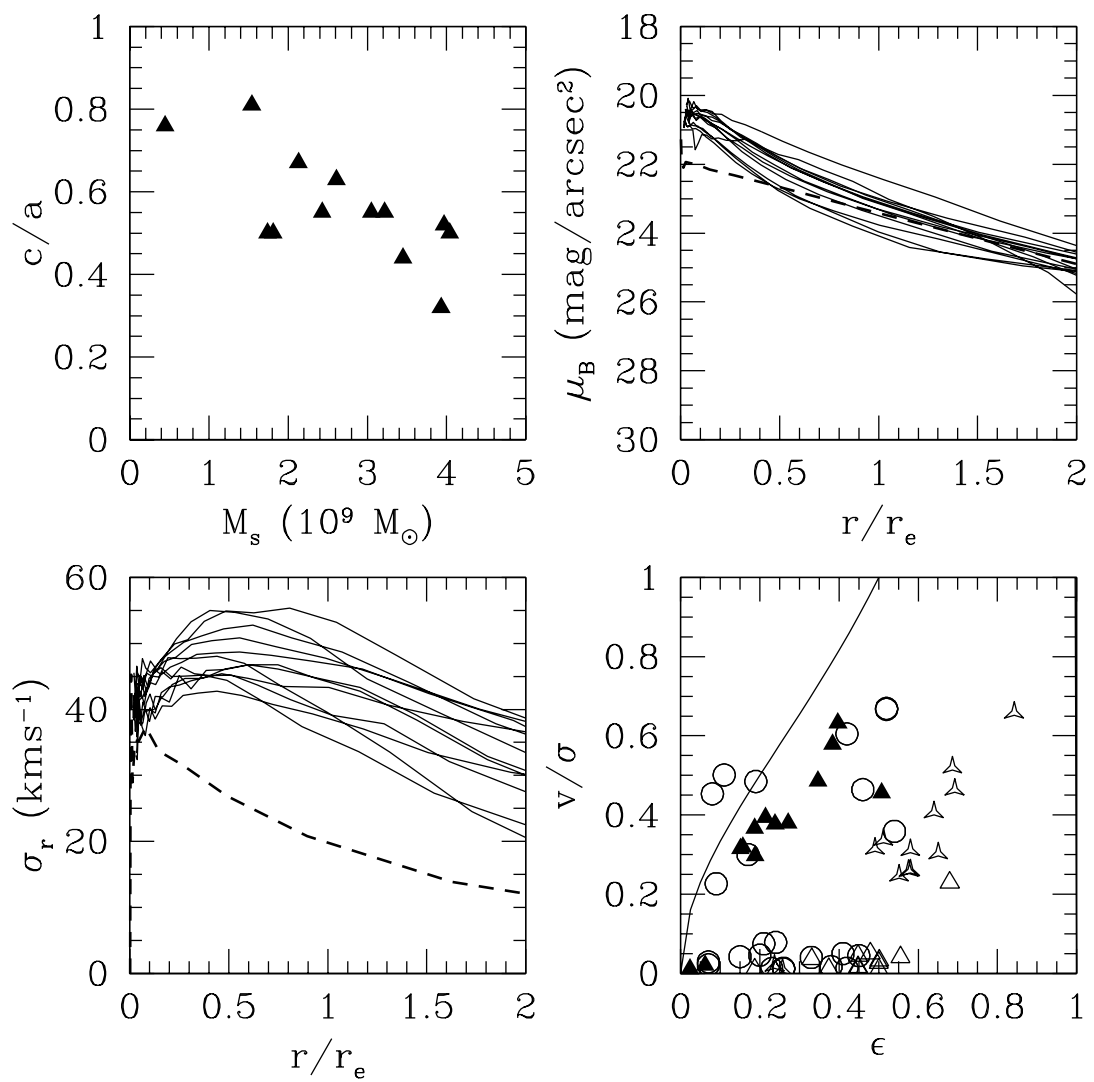

Figure 3. From the top left to the bottom right: final flattening of the remnants plotted against stellar mass, surface brightness and radial velocity dispersion profiles within two scale radii (the thick dashed line represents the initial conditions), ratio of the rotation velocity to the projected velocity dispersion versus the mean ellipticity within $r_{e}$. Open circles represent data from Geha et al. (2002,2003), Pedraz et al. (2002) and Simien \& Prugniel (2002). The results of the simulation are indicated for three different projections along the major (solid triangles), intermediate (deformed triangles) and short axis (open triangles). The solid line shows the theoretical relation for an oblate, isotropic galaxy flattened by rotation.

Geha, M., Guhathakurta, P. \& van der Marel, R. P. 2002 AJ 124, 3073

Geha, M., Guhathakurta, P. \& van der Marel, R. P. 2003 AJ 126, 1794

Graham, A. W., Jerjen, H. \& Guzmán, R. 2003 AJ 126, 1787

Hernquist, L. 1993 ApJ, 86, S389

Jerjen, H., Kalnajs, A. \& Binggeli, B. 2000 A $\& A$ 358, 845

Mayer, L., Governato, F., Colpi, M., Moore, B., Quinn, T., Wadsley, J., Stadel, J. \& Lake, G. 2001 ApJ 559, 754

Moore, B., Katz, N., Lake, G., Dressler, A. \& Oemler, A. 1996 Nature 379, 613

Moore, B., Lake, G. \& Katz, N. 1998 ApJ 495, 139

Moore, B., Lake, G., Quinn, T. \& Stadel, J. 1999 MNRAS 304, 465

Pedraz, S., Gorgas, J., Cardiel, N., Sánchez-Blázquez, P. \& Guzmán, R. 2002, MNRAS 332, L59

Simien, F. \& Prugniel, P. 2002 A $\mathscr{E} A$ 384, 371

Springel, V. \& White, S. D. M. 1999 MNRAS 307, 162

Stadel, J. 2001 Ph.D. Thesis 